\title{
Thermal insulation and hydrophobization of wood impregnated with silica aerogel powder
}

\author{
Hong Chen ${ }^{1 *}$, Yutian Zhang ${ }^{1}$, Tuhua Zhong ${ }^{2}$, Zhihui Wu ${ }^{1}$, Xianxu Zhan ${ }^{3}$ and Jiaoyou Ye ${ }^{3}$
}

\begin{abstract}
The aim of the study was to develop a simple and cost-effective method to improve thermal insulation and hydrophobicity of wood. Herein, we attempted to use commercially available silica aerogel powders suspended in ethanol to treat the wood by a simple vacuum impregnation process. The effects of particle size ( $20 \mu \mathrm{m}$ and $40 \mathrm{~nm})$ of silica aerogels and the number of impregnation cycles (1, 3, and 5 cycles) were examined on the thermal conductivity and the surface hydrophobicity. The results showed that the thermal conductivity of silica aerogel-impregnated wood decreased by approximately $38 \%$. The water contact angle of the impregnated wood increased up to the maximum values $153^{\circ}$ with a comparison with $80^{\circ}$ of the untreated wood, indicating effective hydrophobization after silica aerogel impregnation. The tensile properties of the impregnated wood were found slightly improved. The results indicate that the impregnation of silica aerogel powders in wood can be a facile and efficient approach to prepare wood with thermal insulation and hydrophobicity, which may hold great promise to be employed in manufacturing wood-based materials used in interior decoration and buildings.
\end{abstract}

Keywords: Wood, Silica aerogel powder, Impregnation, Thermal insulation, Hydrophobicity

\section{Introduction}

With the awareness of energy-saving and the sustainability goals, the demand for thermal insulation sustainable products is increasing in building or interior decoration. Wood, a sustainable material, possesses unique visual properties and is friendly to humans and the environment, which has long been used in interior decoration and construction. The thermal conductivity of wood is relatively high (about $0.1 \mathrm{~W} \mathrm{~m}^{-1} \mathrm{~K}^{-1}$ ) [1], but the wood with high thermal insulation is desired in interior decoration and construction applications. Moreover, wood is sensitive to water due to the hydrophilic nature of its main chemical components cellulose and hemicellulose [2], which often leads to low dimension stability of wood or wood-based products. Therefore, searching for a

\footnotetext{
${ }^{*}$ Correspondence: chenhong@nju.edu.cn

${ }^{1}$ College of Furnishings and Industrial Design, Nanjing Forestry University, Nanjing 210037, China

Full list of author information is available at the end of the article
}

simple, cost-effective, and industrially scalable approach is highly needed to enhance thermal insulation and hydrophobicity of wood, especially for the treatment on the less inferior fast-growing wood.

Silica aerogel with ultra-low density and highly crosslinking structure has been regarded as one of the most efficient thermal insulating materials in addition to its excellent flame retardancy [3, 4]. Due to its attractive features of thermal insulation and flame retardancy, silica aerogel has been extensively applied to wood [ 1 , 5, 6], fiber-reinforced composites [7-10], cement-based composites [11, 12], glass materials [13] and other nanocomposites [14]. To date, wood modified with silica aerogels is mainly by means of in situ synthesis $[1,6]$ or impregnation with sol [5]. It was reported that thermal insulation, flame retardancy, and dimensional stability of wood could be significantly improved through in situ polymerization of silica aerogel in the wood cell lumen. The mechanical properties and thermal properties of wood could be enhanced after being impregnated with
Springer Open (c) The Author(s) 2020. This article is licensed under a Creative Commons Attribution 4.0 International License, which permits use, sharing, adaptation, distribution and reproduction in any medium or format, as long as you give appropriate credit to the original author(s) and the source, provide a link to the Creative Commons licence, and indicate if changes were made. The images or other third party material in this article are included in the article's Creative Commons licence, unless indicated otherwise in a credit line to the material. If material is not included in the article's Creative Commons licence and your intended use is not permitted by statutory regulation or exceeds the permitted use, you will need to obtain permission directly from the copyright holder. To view a copy of this licence, visit http://creativeco mmons.org/licenses/by/4.0/. 
silica sol. However, the situ synthesis of silica aerogel in the wood was usually prepared by the sol-gel method via drying under ambient pressure or drying with supercritical $\mathrm{CO}_{2}$. This process is complicated and expensive [4], which lowers its acceptance by manufacturing factories that produce wood-based materials for interior decoration and buildings, such as plywood, laminated veneer lumber (LVL), thus greatly limiting its industrial application.

Silica aerogel powders are easily manufactured and readily available in the market [15]. In this study, we adopted a simple and industrially scalable process, in which commercial silica aerogel powders suspended in ethanol medium were applied directly to the wood through a facile vacuum impregnation, to improve its thermal insulation and hydrophobicity. To our knowledge, there are no reports on directly applying commercial silica aerogel powders to wood. Compared with the complication of in situ polymerization followed by supercritical $\mathrm{CO}_{2}$ drying to form silica aerogel in the wood, the direct impregnation of commercial silica aerogel powders into wood is more straightforward and cost-effective, which makes it more practical in the industry. The microstructure of the impregnated wood was observed by scanning electron microscopy (SEM) to reveal the dispersion of silica aerogel in the wall and lumen of wood cells. The thermal insulation and hydrophobicity of the impregnated wood were measured to examine the influences of particle size of silica aerogel powders and the number of impregnation cycle on the material properties.

\section{Experimental procedure \\ Materials}

Commercially available 20 -mm-thick veneers with density at $0.428 \pm 0.044 \mathrm{~g} \mathrm{~cm}^{-3}$ made of fast-growing poplar (Populus L.) were provided by Dehua Tubao New Decoration Material Co., Ltd., Huzhou, Zhejiang Province, China. Silica aerogel powders with an average diameter of $40 \mathrm{~nm}$ and $20 \mu \mathrm{m}$, respectively, were purchased from Langfang Taoge Co., Ltd., Langfang, Hebei Province, and Suzhou Tanfeng Co., Ltd., Suzhou, Jiangsu Province, China. The morphology of these two powders and photographs of the water droplet on their surface are shown in Fig. 1. The super-lightweight silica aerogel powders with an average diameter of $40 \mathrm{~nm}$ cannot firmly sustain a water droplet, the water droplet was partly enclosed by the powders, but water did not diffuse and penetrate into the silica aerogel, indicating its excellent hydrophobicity. The thermal conductivity of silica aerogel powder with average diameter at $40 \mathrm{~nm}$ and $20 \mu \mathrm{m}$ was $0.042 \mathrm{~W} \mathrm{~m}^{-1}$ $\mathrm{K}^{-1}$ and $0.056 \mathrm{~W} \mathrm{~m}^{-1} \mathrm{~K}^{-1}$, respectively.
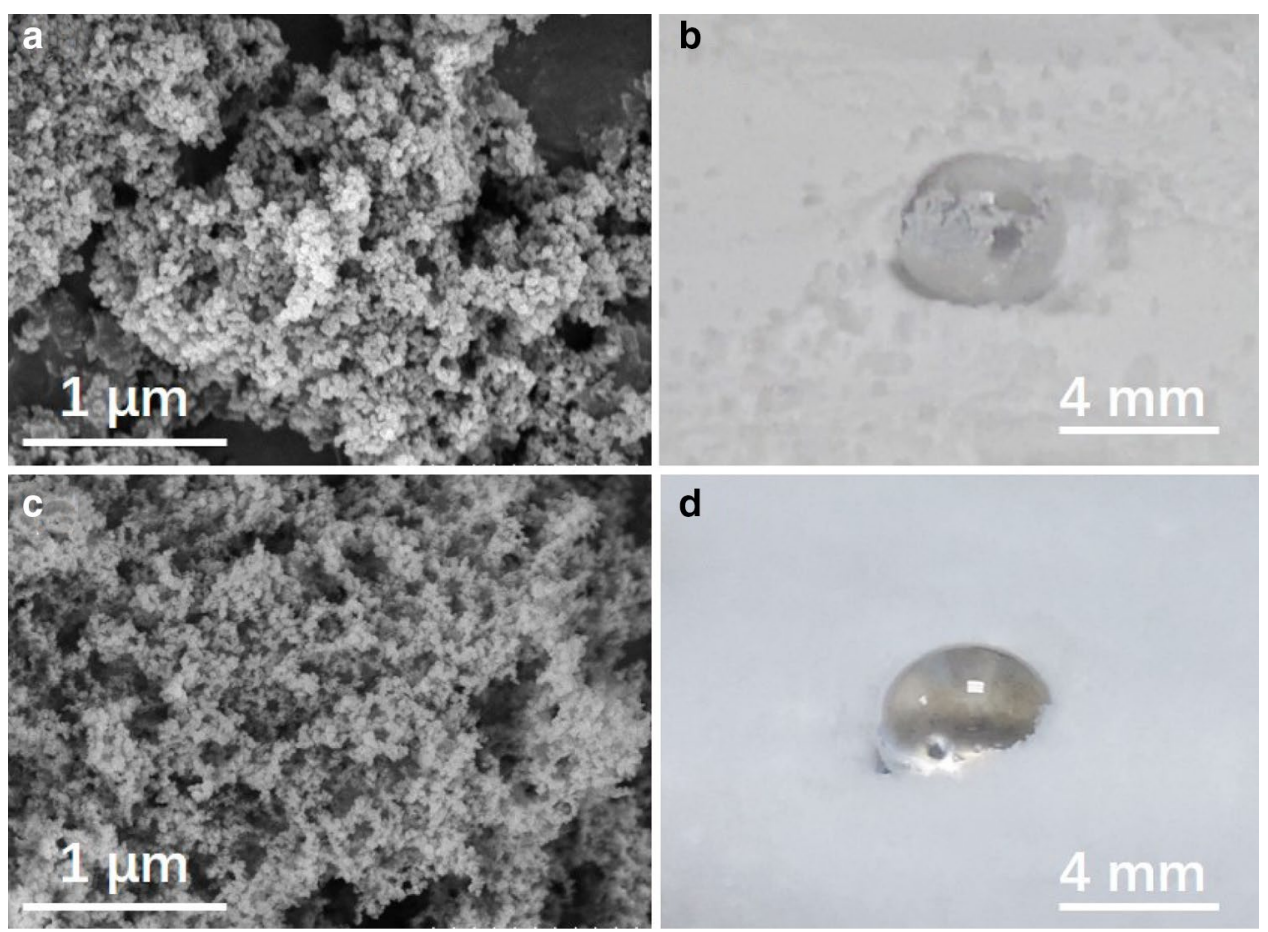

Fig. 1 The ESEM images and silica aerogel powders and the photographs of water droplet on silica aerogel. a, b 20- $\mu$ m size, c and $\mathbf{d} 40-\mathrm{nm}$ size 


\section{Silica aerogel-impregnated wood process}

The silica aerogel powders were first suspended in ethanol to get a suspension with a consistency of $0.1 \mathrm{wt} \%$ and $0.05 \mathrm{wt} \%$, respectively. The wood veneer was air-dried and then was cut into a small specimen with a dimension of $10 \mathrm{~cm} \times 10 \mathrm{~cm}$. Subsequently, the small wood veneer specimens were immersed in silica aerogel powder suspension in a sealed tank. The impregnation of silica aerogel into the wood was facilitated under reduced internal pressure of the tanker by the vacuum pump. The internal pressure was reduced to $-0.09 \mathrm{MPa}$, and then the impregnation was held for $3 \mathrm{~h}$ as well as the vacuum of the tank. Upon the completion of impregnation, the wet impregnated wood veneer specimens were drying at $103{ }^{\circ} \mathrm{C}$ in an oven for $2 \mathrm{~h}$. The impregnation treatment was conducted repeatedly with various cycles $(1,3$, and 5 cycles). The processing parameters and the schematic diagram of the impregnation are shown as Table 1 and Fig. 2, respectively.

Table 1 Sample codes and the corresponding processing parameters

\begin{tabular}{llll}
\hline Treatments & Average diameter & $\begin{array}{l}\text { Concentration } \\
\text { (wt\%) }\end{array}$ & Cycles \\
\hline S-40-1 & $40 \mathrm{~nm}$ & 0.1 & 1 \\
S-40-3 & & & 3 \\
S-40-5 & & 5 \\
S-20-1 & $20 \mu \mathrm{m}$ & 0.05 & 1 \\
S-20-3 & & 3 \\
S-20-5 & & 5 \\
\hline
\end{tabular}

\section{Characterizations}

The weight of sample treated and untreated were measured and the weight grain rate was calculated according to the Formula (1):

$$
\text { Weight grain rate }=\frac{\text { weight after treatment }- \text { weight before treatment }}{\text { weight before treatment }} \times 100 \%
$$

The cross-section of wood veneer was cut from the center of both modified and unmodified samples. The surface and cross-section of modified and unmodified wood veneers were covered by gold and then observed with an emission scanning electron microscope (ESEM) and energy dispersive spectrometer (EDS) (Quanta 200, FEI Company, USA). The water contact angles of modified and unmodified wood veneers were measured with an optical contact angles instrument (JC2000C1, China) with room temperature at about $25^{\circ} \mathrm{C}$ and related humidity at $55 \%$. The water droplet volume was $2 \mu \mathrm{L}$. The water contact angles were calculated using the ellipse method. Six replicates were tested for each sample. Wood veneers were cut into $30 \times 30 \mathrm{~mm}$ test specimens for thermal conductivity measurement. The thermal conductivity of wood was measured with a thermal conductivity tester (TCI-2-A, C-Therm, Canada) with room temperature at $15{ }^{\circ} \mathrm{C}$. Three replicates were measured for each type. Wood veneers were cut into a strip with a dimension of $100 \mathrm{~mm}$ longitudinally and $10 \mathrm{~mm}$ in width for tensile testing. Both ends of the samples were covered by wood veneer for avoiding been compressed to broke by the mechanical tester. The tensile testing of wood veneer specimens was performed by a commercial mechanical

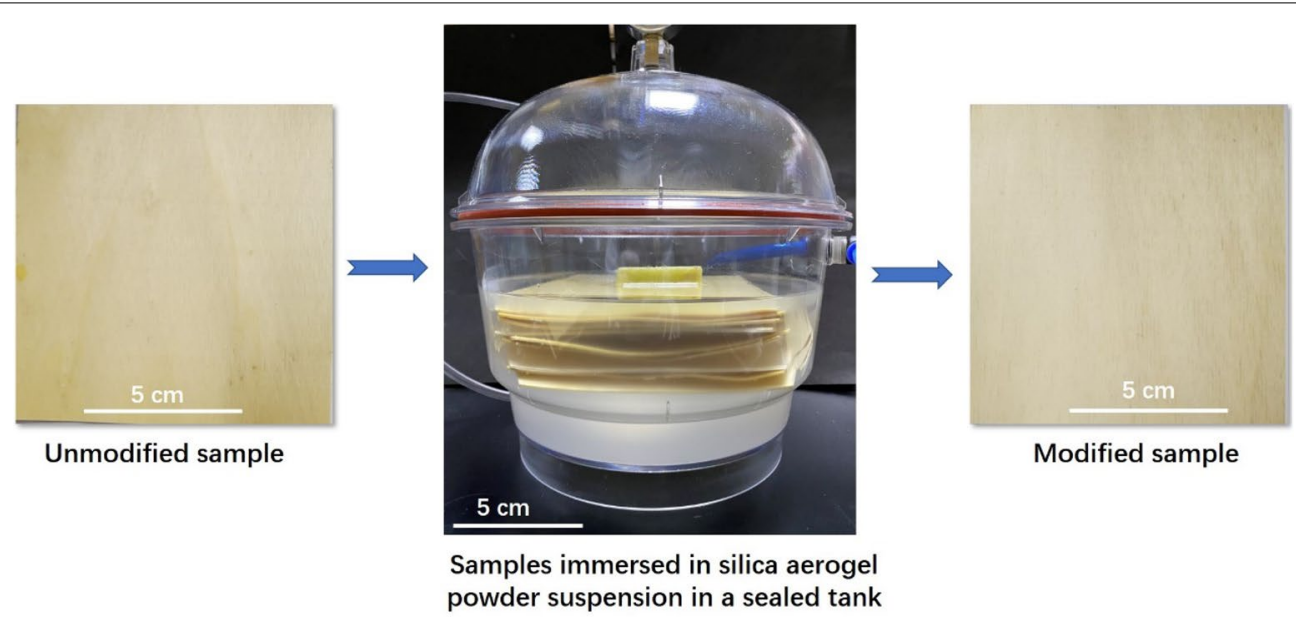

Fig. 2 The schematic diagram of impregnation 


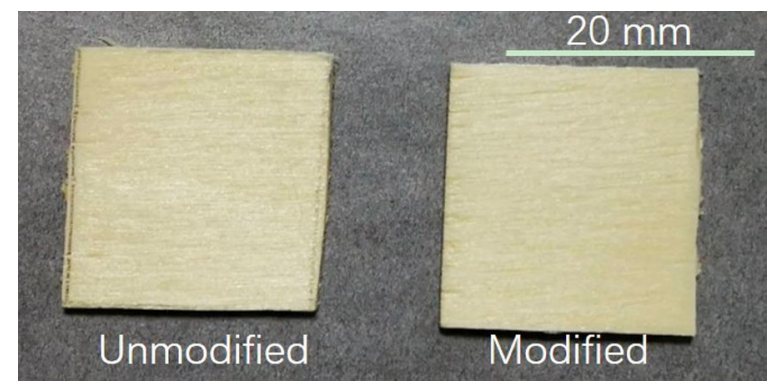

Fig. 3 The unmodified and modified wood veneers

Table 2 The weight gain rate of modified wood

\begin{tabular}{lll}
\hline Samples & Weight gain rate (\%) & $\begin{array}{l}\text { Standard } \\
\text { deviation }\end{array}$ \\
\hline S-40-1 & 1.14 & 0.32 \\
S-40-3 & 0.35 & 0.26 \\
S-40-5 & 0.54 & 0.23 \\
S-20-1 & 1.28 & 0.27 \\
S-20-3 & 0.32 & 0.18 \\
S-20-5 & 0.47 & 0.26 \\
\hline
\end{tabular}

tester (AG-IC100KN, Shimadzu, Japan) with a strain rate of $1.0 \mathrm{~mm} / \mathrm{min}$. Six specimens were tested for each type.

\section{Results and discussion}

\section{The microstructure and chemical components of modified} wood

Figure 3 shows the surface of both unmodified and modified wood veneers. The impregnation of silica aerogel barely altered the visual properties of wood, which is of great importance for wood-based materials intended for the use in interior decoration.

The weight gain rate of wood veneer is shown in Table 2 . The weight gain rate was mainly determined by the impregnation cycles. The weight gain rate of samples modified in 1 cycle was the highest among all the modified samples. When increased to 3 and 5 cycles, the weight gain rate sharply decreased, it was possibly because the extractives in wood were extracted by ethanol. The removal of extractives compromised the weight gain of wood. The microstructure and the element silica (Si) distribution on the surface and cross-section of wood veneers are shown in Figs. 4, 5, 6. There was almost no Si element found in the unmodified wood, as evidenced by EDS elemental mapping analysis in Fig. $4 \mathrm{~b}$ and d. The element silica existed and was distributed on the surface and the cross-section of the silica aerogel-impregnated wood because the red dots that represented element $\mathrm{Si}$ were observed in the Si element map (Figs. 5 and 6). This indicated that silica aerogel was successfully impregnated in the wood cells. When the impregnation cycles increased, the number of red dots increased and thus red dots became brighter because of the increase in the silica concentration on the surface and cross-section of the impregnated wood. This is consistent with the EDS silica quantitative analysis as summarized in Table 3 . The weight fraction and atomic fraction of the element $\mathrm{Si}$ in the wood impregnated with large silica aerogel powders ( $20 \mu \mathrm{m}$ in diameter) were higher than those in the wood impregnated with small ones ( $40 \mathrm{~nm}$ in diameter). It was also indicated in the magnified images of Fig. 7 that with the increasing impregnation cycles, the amount of silica aerogel in samples increased.

\section{Thermal conductivity of wood}

The thermal conductivity of modified and unmodified wood is shown in Fig. 8. The conductivity of the silica aerogel-impregnated wood decreased compared with the unmodified wood. The decrease of thermal conductivity was dependent on the number of the impregnation cycles and the diameter of silica aerogel powders. For the small silica aerogel powders ( $40 \mathrm{~nm}$ in diameter), the thermal conductivity of the wood impregnated with 1,3 , and 5 cycles decreased by $11.2 \%, 11.5 \%$, and $38.1 \%$, respectively. For the large $20-\mu \mathrm{m}$ silica aerogel powder, the thermal conductivity decreased by $14.0 \%, 27.0 \%$, and $33.1 \%$, respectively. It appears that the thermal conductivity of all the wood impregnated directly with aerogel powders decreased more effectively than that of wood modified by the in situ synthesis of silica aerogel through the sol-gel method with supercritical $\mathrm{CO}_{2}$ drying [1]. By comparison, the thermal conductivity of wood treated with S-405, S-20-3 and S-20-5 decreased significantly, especially that of wood treated with S-40-5 and S-20-5. The direct impregnation of $20-\mu \mathrm{m}$ silica aerogel powders into the wood for 3 cycles might be considered as the sufficient and the most cost-effective treatment, given the cost of impregnation and its efficiency of decreasing thermal conductivity of wood.

\section{The hydrophobicity of wood}

Figure 9 shows the water contact angles of unmodified and modified wood. The water contact angles of silica aerogel-impregnated wood were significantly increased in comparison with the untreated wood. The impregnation cycle played an important role in influencing the hydrophobicity of the impregnated wood. One-cycle impregnation of $40-\mathrm{nm}$ and $20-\mu \mathrm{m}$ silica aerogel powders brought the water contact angle up to $100^{\circ}$ and $110^{\circ}$, respectively, compared to approximately $80^{\circ}$ for the untreated wood. When the impregnation cycles increased to three times, the water contact angles of the 

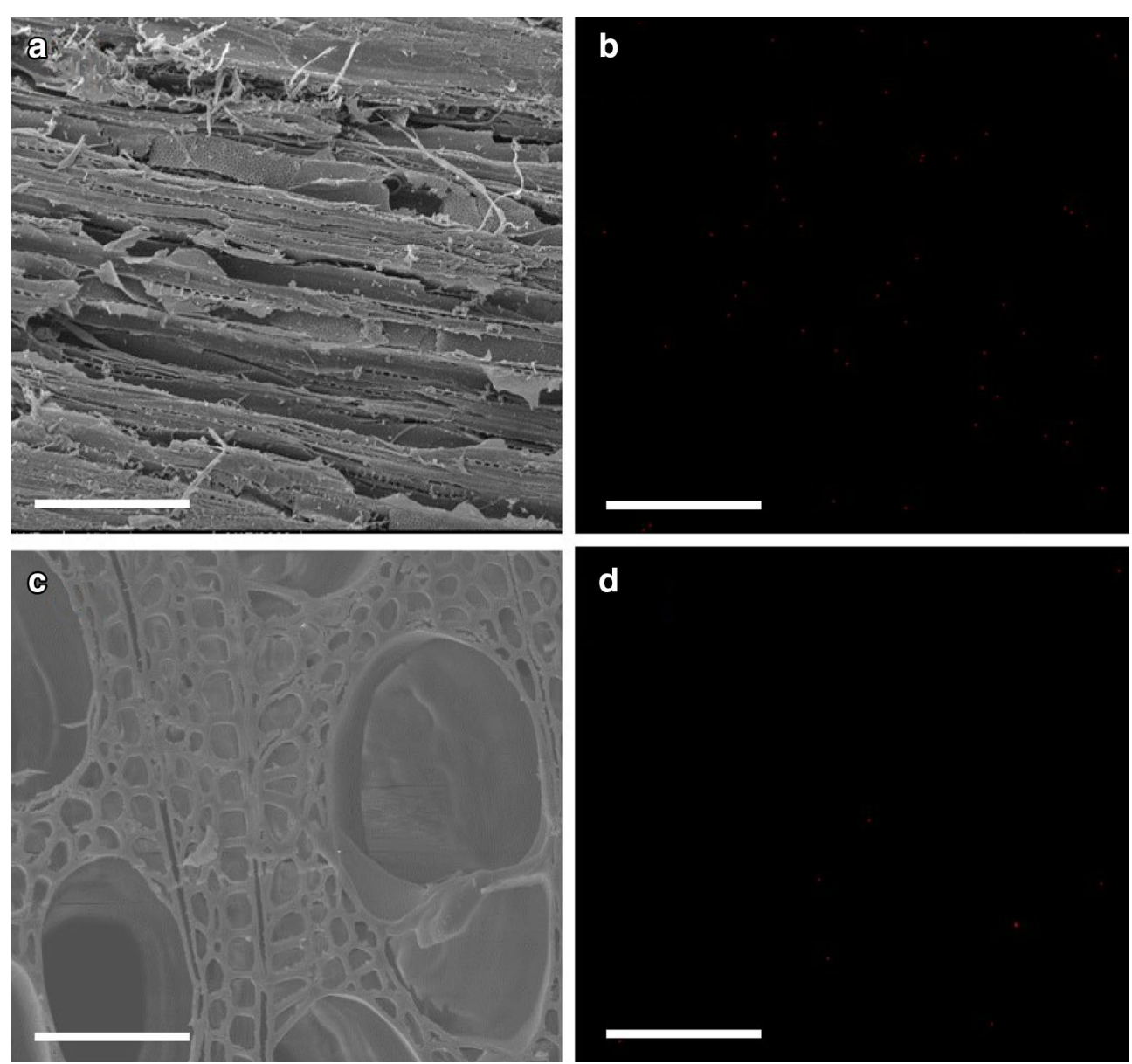

Fig. 4 ESEM images of the surface (a) and the cross-section (c) of the unmodified wood, and the corresponding EDS elemental mapping of Si on the surface (b) and the cross-section (d), respectively. The scale bar is $100 \mu \mathrm{m}$

impregnated wood increased to the maximum values $153^{\circ}$ for S-40-3 sample and $148^{\circ}$ for S-20-3 sample, which increased by $65.0 \%$ and $63.1 \%$, respectively. There was a remarkable increase in the water contact angle after a three-cycle impregnation because repeated impregnation resulted in more accumulated silica aerogels on the surface of the impregnated wood when compared to one-cycle impregnation. Thus the wood surface was more effectively covered with hydrophobic silica aerogel powders. It is interesting to note that further impregnation (5 cycles) slightly decreased the water contact angles and decreased the adhesion of the water droplet on the wood surface (Additional file 1: Video S1). Additional file 1: Video S1 indicated that the water droplet was easier to slide on the wood surface modified with more impregnation cycles. In this study, all silica aerogel powder-impregnated wood changed from a hydrophilic surface to a much more hydrophobic one. In the previous research [5], the water contact angle of wood impregnated with silica sol merely increased from about 65 to $85^{\circ}$. It indicated that the direct impregnation of silica aerogel powders into wood was much more effective in the hydrophobization of wood than the impregnation of silica sol.

The adhesion of silica aerogel to the wood surface was assessed by a washing process. The change in the water contact angles of the impregnated wood with or without washing by pouring ethanol is shown in Fig. 10. There is no significant change in the water contact angles of the 40-nm silica aerogel-impregnated wood with or without the washing process, while the water contact angle of the $20-\mu \mathrm{m}$ silica aerogel-impregnated wood decreased after the washing; it suggested that smaller silica aerogel powders had better adhesion to the wood than the large ones.

As shown in Fig. 11, water droplets stood steadily on the treated wood surface, but penetrated into the untreated wood surface. The wood modified with S-40-3 and S-40-5 was not only superhydrophobic, but also showed self-cleaning properties, which was demonstrated by dropping water to the carbon 


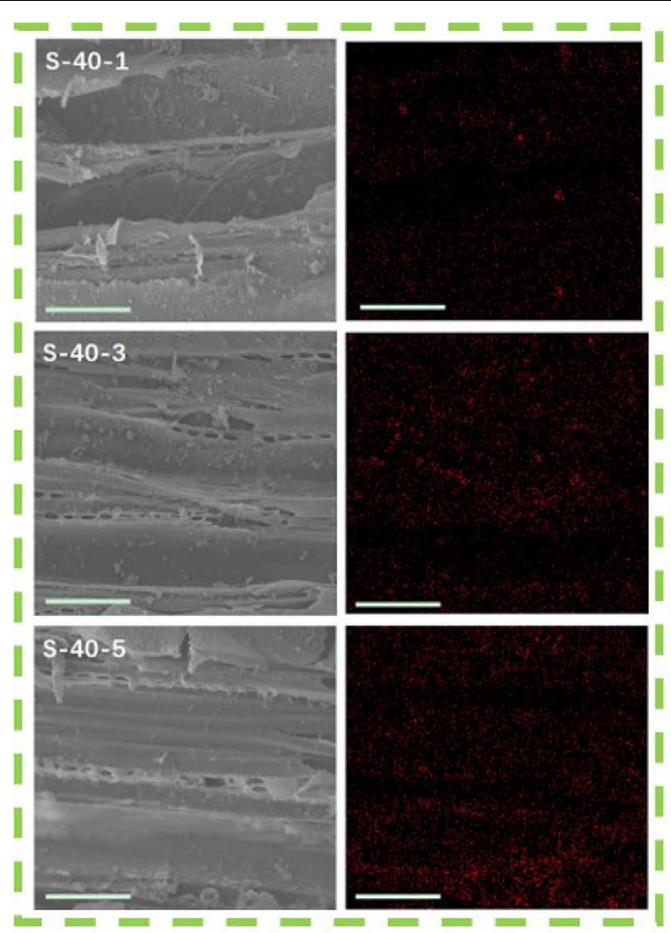

a Surface

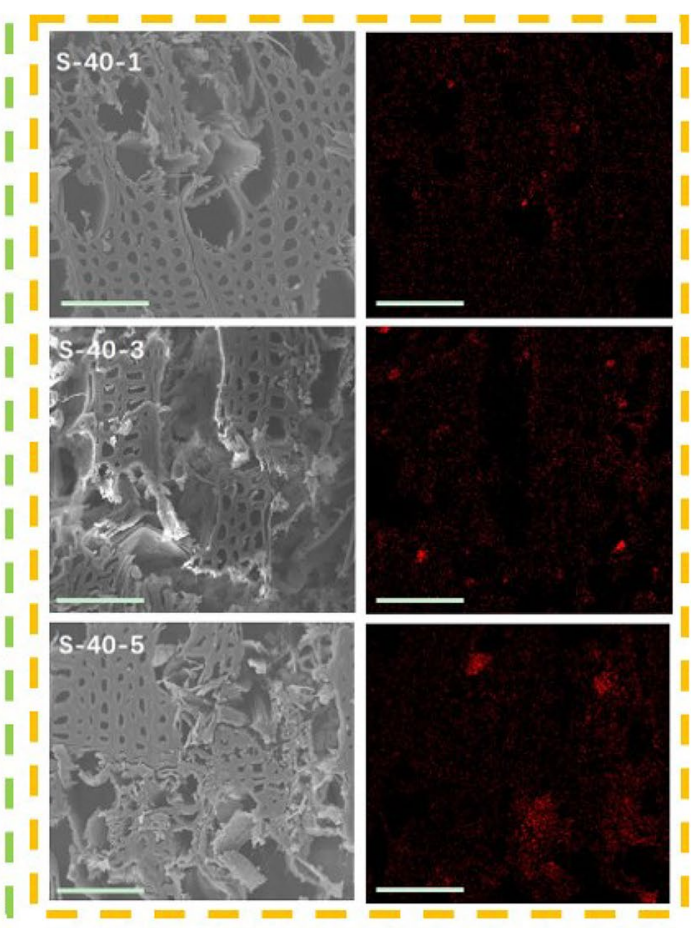

b Cross-section

Fig. 5 ESEM images showing the microstructure (left) and the corresponding Si element mapping (right) of the surface (a) and the cross-section (b) of wood modified by silica aerogel powders with an average diameter of $40 \mathrm{~nm}$. The scale bar is $100 \mu \mathrm{m}$
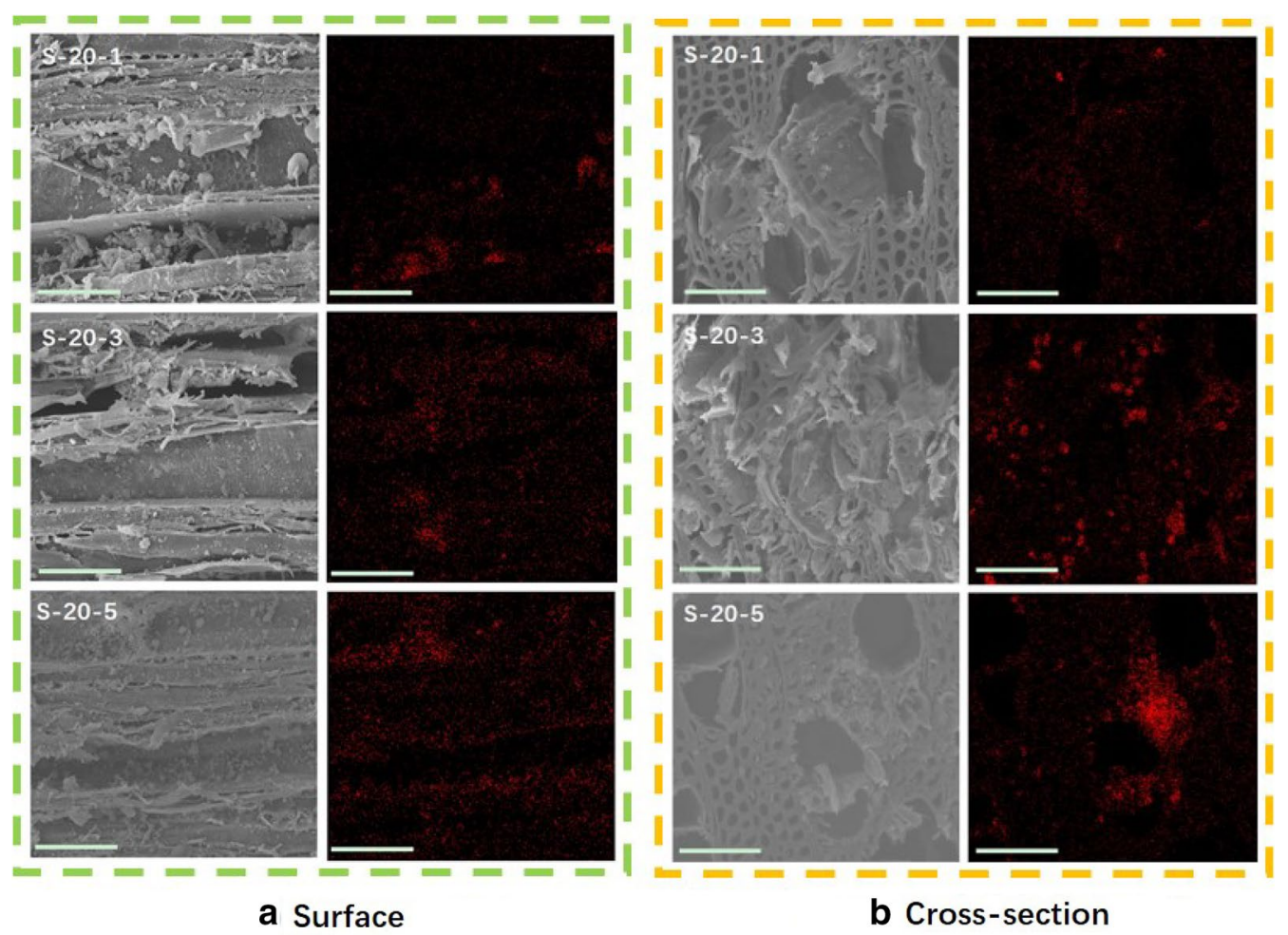

Fig. 6 ESEM images showing the microstructure (left) and the corresponding Si element mapping (right) of the surface (a) and the cross-section (b) of wood modified by silica aerogel powders with an average diameter of $20 \mu \mathrm{m}$. The scale bar is $100 \mu \mathrm{m}$ 
Table 3 The weight fraction and atomic fraction of Si in the surface and cross-section of the unmodified and modified wood

\begin{tabular}{llllll}
\hline Samples & \multicolumn{2}{l}{ Surface } & & & \multicolumn{2}{l}{ Cross-section } \\
\cline { 2 - 3 } \cline { 5 - 6 } & Weight (\%) & Atomic (\%) & & Weight (\%) & Atomic (\%) \\
\hline Control & 0.12 & 0.06 & & 0.01 & 0.01 \\
S-40-1 & 1.90 & 0.97 & & 1.77 & 0.90 \\
S-40-3 & 3.84 & 1.98 & & 2.31 & 1.18 \\
S-40-5 & 4.65 & 2.42 & & 5.34 & 2.78 \\
S-20-1 & 4.52 & 2.32 & & 1.30 & 0.66 \\
S-20-3 & 6.98 & 3.64 & 6.01 & 3.13 \\
S-20-5 & 8.26 & 4.37 & 7.83 & 4.15 \\
\hline
\end{tabular}

powder-contaminated wood surface. From the perspective of obtaining an effective and durable hydrophobic surface, smaller silica aerogel powders are more ideal for the hydrophobization of wood.

\section{Tensile properties}

The tensile strength of the unmodified and modified wood is shown in Fig. 12. Overall, the impregnation of silica aerogel into wood increased the tensile strength to varying extents, which was mainly determined by the number of the impregnation cycles. It was found that one-cycle impregnation of 40-nm silica aerogel powders significantly increased the tensile strength by $86.6 \%$, the increase was much higher that $17.4 \%$ for the wood impregnated with $20-\mu \mathrm{m}$ aerogel powders. This might be attributed to better adhesion of smaller silica aerogel to the wood, thus providing the reinforcing effect. In addition, the $40-\mathrm{nm}$ silica aerogel powder was easier to fill the small pores in wood than $20-\mu \mathrm{m}$ silica aerogel powder, which also lead to a high tensile property. Besides, the fracture forms of modified wood and unmodified wood were different, as shown in Fig. 13. The unmodified wood was fragile and readily broken by a tensile stress, while the modified wood was tougher and not easy to be broken. This is likely due to that the silica aerogel powders filled the parts of pores in cell wall such as pits or lumen, which improved the mechanical properties [5]. By contrast, it was reported that the tensile strength of the wood modified by the in situ synthesis of silica aerogel decreased in comparison with the untreated wood. This was mainly ascribed to the microcracks in the cell wall, which was generated by the effect of chemical solutions [1]. It seems that the direct impregnation of silica aerogel powders suspended in ethanol via a simple vacuum impregnation was a more gentle means than the formation of silica aerogels by means of the in situ synthesis, which might not impair wood cells and therefore lead to a positive effect on the mechanical properties.

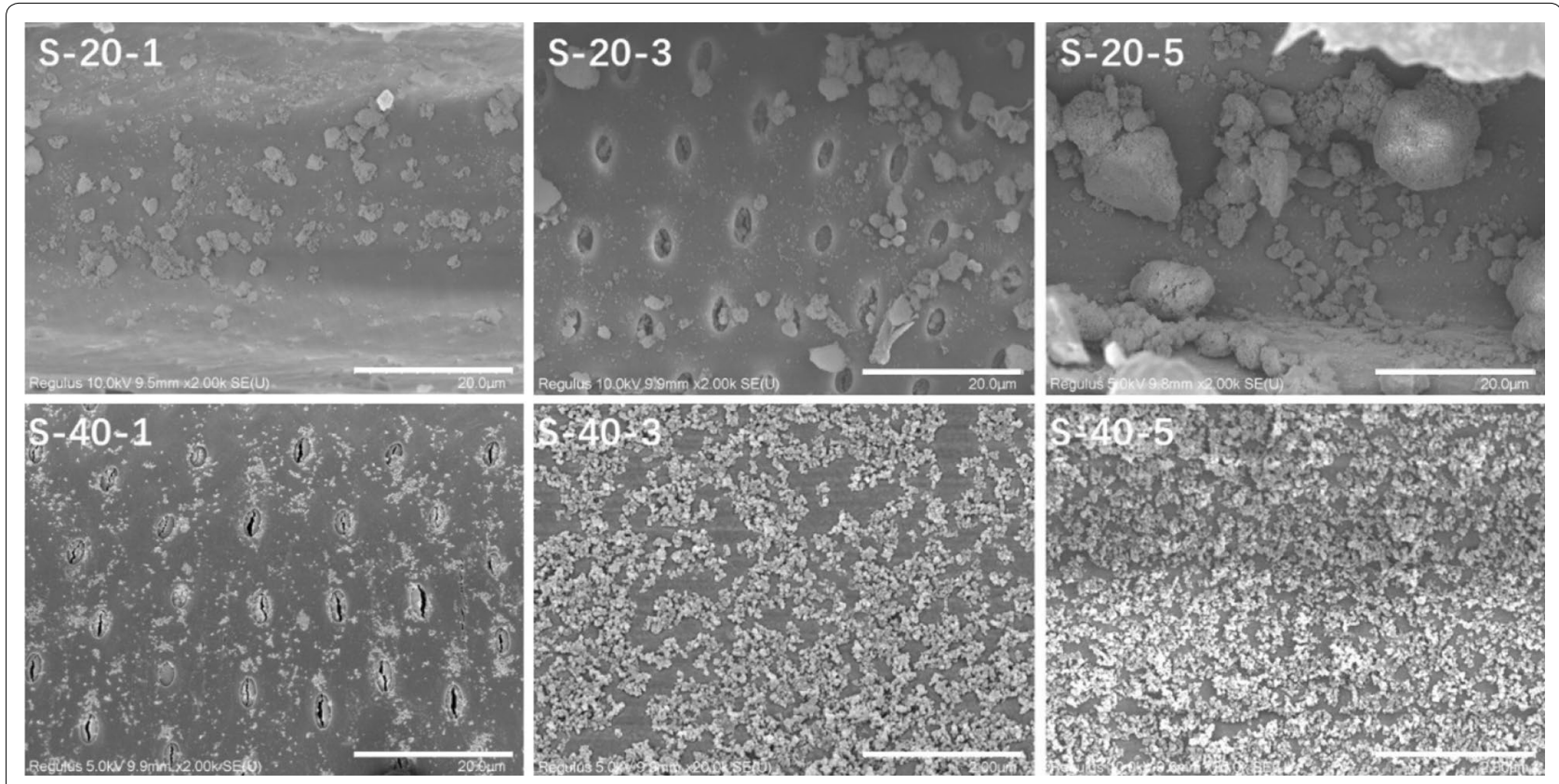

Fig. 7 Magnified ESEM images of the silica aerogel powder on the surface of modified samples. The scale bar is $20 \mu \mathrm{m}$ 


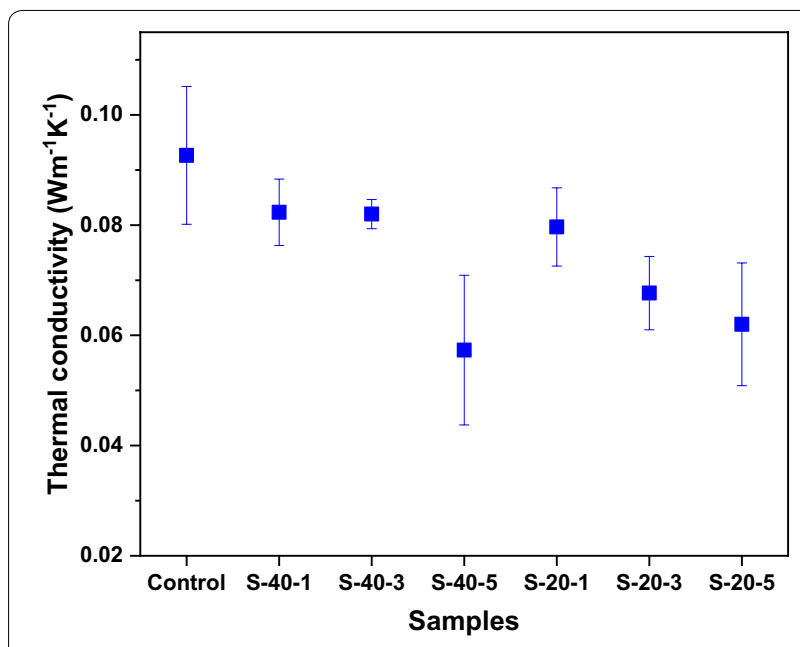

Fig. 8 The thermal conductivity of unmodified and modified wood

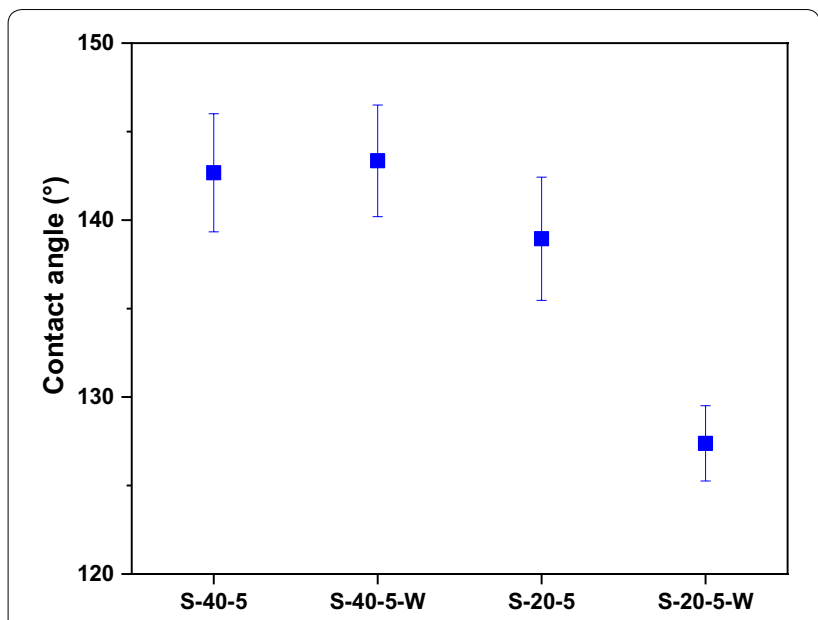

Fig. 10 The contact angles of the modified wood with or without the washing process

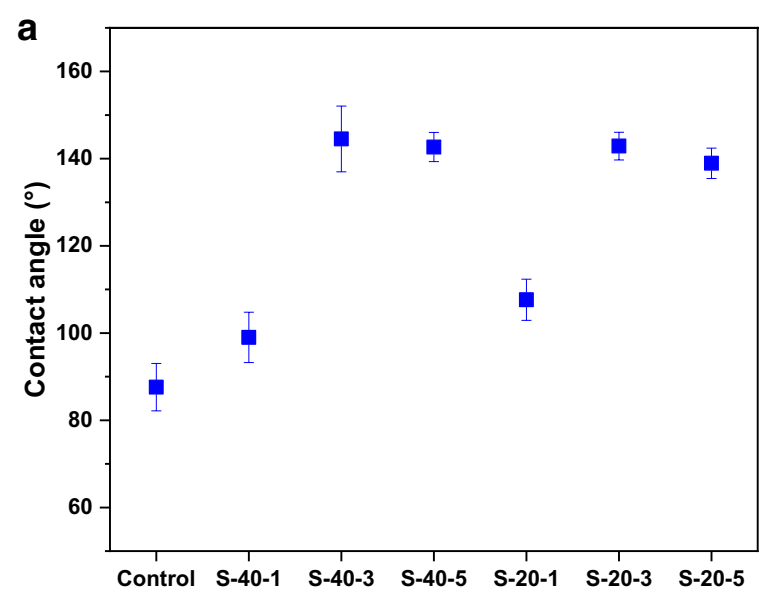

b

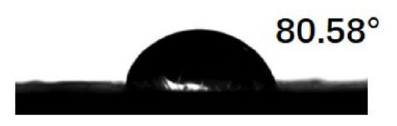

Control

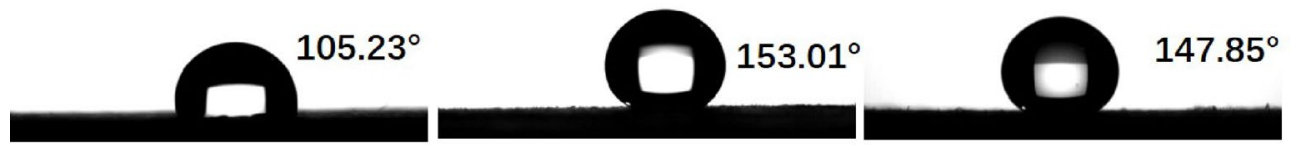

S-40-1

S-40-3

S-40-5
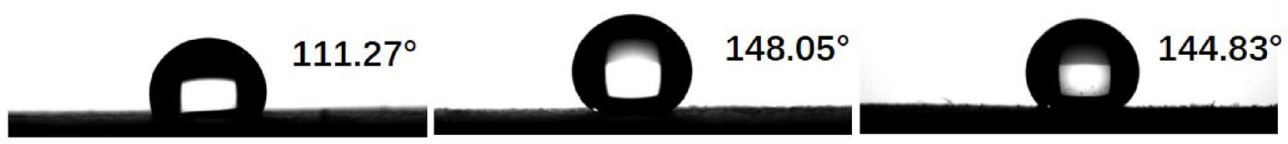

S-20-1

S-20-3

S-20-5

Fig. 9 The water contact angles of unmodified and modified wood. a Water contact angles, $\mathbf{b}$ the image of water droplet on wood surface 


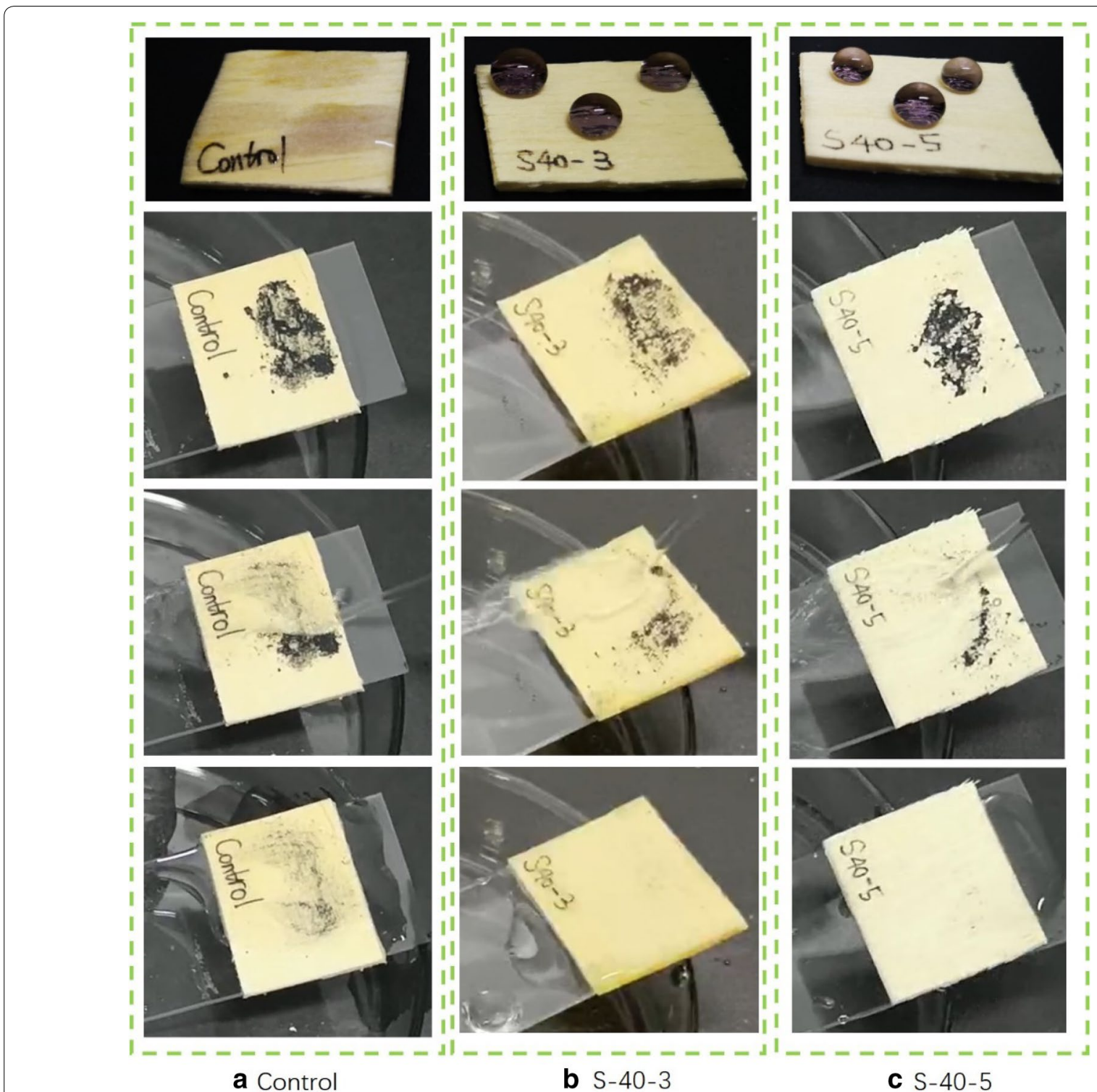

Fig. 11 The water droplet on the wood surface and self-cleaning process on a untreated wood, $\mathbf{b}$ wood treated by S-40-3 and $\mathbf{c}$ wood treated by S-40-5 


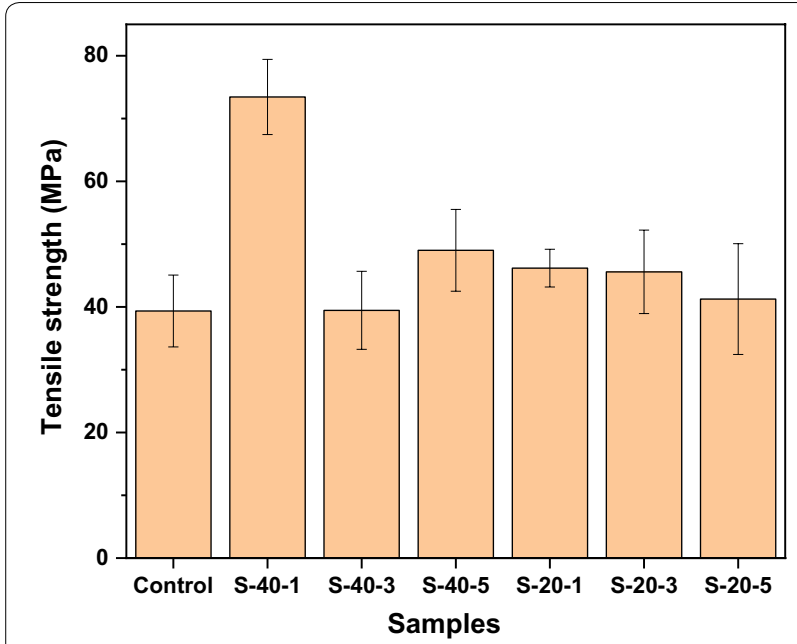

Fig. 12 The tensile strength of unmodified and modified wood

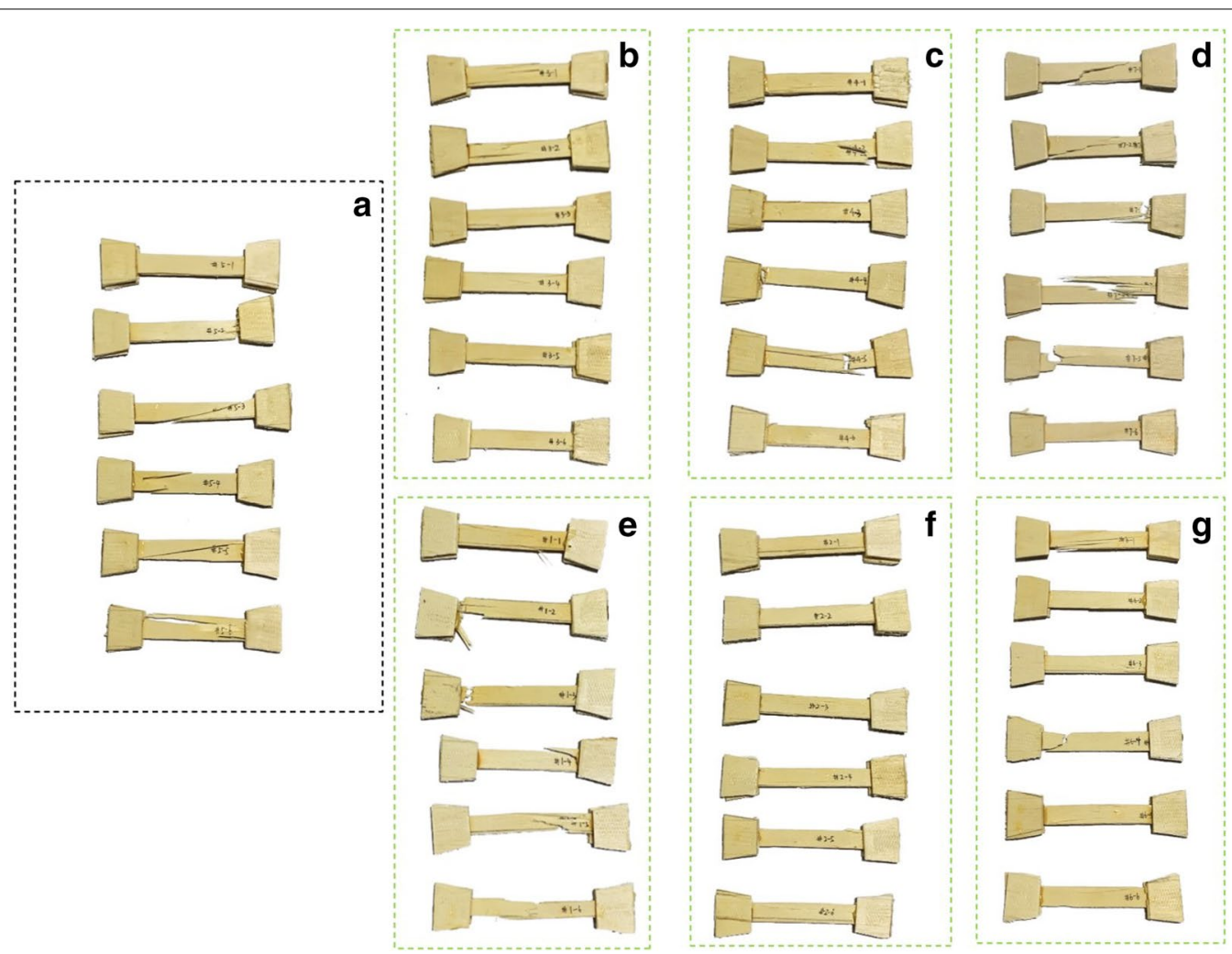

Fig. 13 The fracture form of a unmodified wood and wood modified by b S-40-1, c S-40-3, d S-40-5, e S-20-1, f S-20-3, g S-20-5 


\section{Conclusions}

We presented a novel and industrially scalable method to improve the thermal insulation and hydrophobicity of fast-growing wood by the incorporation of commercially available silica aerogels in the wood through a simple vacuum impregnation process. The number of the impregnation cycles and the particle size of the commercial silica aerogel powders influenced the thermal conductivity and hydrophobicity of the impregnated wood to different degrees. The most cost-effective treatment for obtaining modified wood with good thermal insulation opted for the impregnation of $20-\mu \mathrm{m}$ silica aerogel powders for $3 \mathrm{~h}$ and three impregnation cycles, which provided lower thermal conductivity as well as a much more hydrophobic surface in comparison with the untreated wood. If only considering the hydrophobicity of wood, the best option might be the impregnation of smaller silica aerogel powders with a diameter of $40 \mathrm{~nm}$ for three impregnation cycles, because the adhesion of smaller silica aerogel powders to the wood surface was higher than that of $20-\mu \mathrm{m}$ silica aerogel powders.

\section{Supplementary information}

Supplementary information accompanies this paper at https://doi. org/10.1186/s10086-020-01927-7.

Additional file 1: Video S1. The water repellency of wood modified by silica aerogel.

\section{Abbreviations \\ S-40-1: Modified with 40-nm silica aerogel power in 1-cycle impregnation; S-40-3: Modified with 40-nm silica aerogel power in 3-cycle impregnations; S-40-5: Modified with 40-nm silica aerogel power in 5-cycle impregnations; S-40-5-W: Modified with 40-nm silica aerogel power in 5-cycle impregnations with ethanol washing after each impregnation; S-20-1: Modified with 20- $\mu \mathrm{m}$ silica aerogel power in 1-cycle impregnation; S-20-3: Modified with 20- $\mu \mathrm{m}$ silica aerogel power in 3-cycle impregnations; S-20-5: Modified with 20- $\mu \mathrm{m}$ silica aerogel power in 5-cycle impregnations; S-20-5-W: Modified with 20- $\mu \mathrm{m}$ silica aerogel power in 5-cycle impregnations with ethanol washing after each impregnation; ESEM: Emission scanning electron microscope; EDS: Energy dispersive spectrometer.}

\section{Acknowledgements}

The authors appreciate Jiangjing Shi and Jieyu Wu from Nanjing Forestry University to help prepare the samples, test the tensile properties and record the video.

\section{Authors' contributions}

$\mathrm{HC}$ designed the experiments and was a major contributor in data analysis and writing the manuscript. YZ partly analyzed the data and was a major contributor in conducting the experiments. TZ partly analyzed the data and wrote the manuscript. ZW and XZ financed the research. JY helped $Y Z$ conduct part of experiments. All authors read and approved the final manuscript.

\section{Funding}

This research was supported Postdoctoral Research Foundation of China (2020T130079), Postdoctoral Research Foundation of Zhejiang Province (221478) and National Natural Science Foundation of China (32071687).

\section{Availability of data and materials}

All data generated or analyzed during this study are included in this published article.

\section{Competing interests}

The authors declare that they have no competing interests.

\section{Author details}

${ }^{1}$ College of Furnishings and Industrial Design, Nanjing Forestry University, Nanjing 210037, China. ${ }^{2}$ Composite Materials and Engineering Center, Washington State University, Pullman, WA 99164, USA. ${ }^{3}$ Dehua Tubao New Decoration Material Co., Ltd, Huzhou 313200, China.

Received: 20 August 2020 Accepted: 5 November 2020

Published online: 23 November 2020

\section{References}

1. Sedighi Gilani M, Zhao S, Gaan S, Koebel MM, Zimmermann T (2016) Design of a hierarchically structured hybrid material: via in situ assembly of a silica aerogel into a wood cellular structure. RSC Adv 6:62825-62832

2. Eder M, Schäffner W, Burgert I, Fratzl P (2020) Wood and the activity of dead tissue. Adv Mater 2001412:1-15

3. He YL, Xie T (2015) Advances of thermal conductivity models of nanoscale silica aerogel insulation material. Appl Therm Eng 81:28-50

4. Soleimani Dorcheh A, Abbasi MH (2008) Silica aerogel; synthesis, properties and characterization. J Mater Process Technol 199:10-26

5. Xu E, Zhang Y, Lin L (2020) Improvement of mechanical, hydrophobicity and thermal properties of Chinese fir wood by impregnation of nano silica sol. Polymers 12:1632

6. Zhu X, Wu Y, Tian C, Qing Y, Yao CH (2014) Synergistic effect of nanosilica aerogel with phosphorus flame retardants on improving flame retardancy and leaching resistance of wood. J Nanomater, article ID 867106, 8 pages

7. Lee KJ, Choe YJ, Kim YH, Lee KJ, Hwang HJ (2018) Fabrication of silica aerogel composite blankets from an aqueous silica aerogel slurry. Ceram Int 44:2204-2208

8. Li Z, Gong L, Cheng X, He S, Li CC, Zhang HP (2016) Flexible silica aerogel composites strengthened with aramid fibers and their thermal behavior. Mater Des 99:349-355

9. Li C, Cheng X, Li Z, Pan YH, Huang YJ, Gong LL (2017) Mechanical, thermal and flammability properties of glass fiber film/silica aerogel composites. J Non Cryst Solids 457:52-59

10. Jaxel J, Markevicius G, Rigacci A, Budtova T (2017) Thermal superinsulating silica aerogels reinforced with short man-made cellulose fibers. Compos Part A Appl Sci Manuf 103:113-121

11. Zeng Q, Mao T, Li H, Peng Y (2018) Thermally insulating lightweight cement-based composites incorporating glass beads and nanosilica aerogels for sustainably energy-saving buildings. Energy Build 174:97-110

12. Liu S, Zhu K, Cui S, Shen XD, Tan G (2018) A novel building material with low thermal conductivity: rapid synthesis of foam concrete reinforced silica aerogel and energy performance simulation. Energy Build 177:385-393

13. Cotana F, Pisello AL, Moretti E, Buratti C (2014) Multipurpose characterization of glazing systems with silica aerogel: in-field experimental analysis of thermal-energy, lighting and acoustic performance. Build Environ 81:92-102

14. Cai J, Liu S, Feng J, Kimura S, Wada M, Kuga S, Zhang LN (2012) Cellulosesilica nanocomposite aerogels by in situ formation of silica in cellulose gel. Angew Chemie 124:2118-2121

15. Koebel MM, Huber L, Zhao S, Malfait WJ (2016) Breakthroughs in costeffective, scalable production of superinsulating, ambient-dried silica aerogel and silica-biopolymer hybrid aerogels: from laboratory to pilot scale. J Sol Gel Sci Technol 79:308-318

\section{Publisher's Note}

Springer Nature remains neutral with regard to jurisdictional claims in published maps and institutional affiliations. 